\title{
ON A NILPOTENT LIE SUPERALGEBRA WHICH GENERALIZES $Q_{n}$
}

\author{
José María ANCOCHEA BERMÚDEZ \\ and Otto Rutwig CAMPOAMOR
}

\begin{abstract}
In [6] and [7] the author introduces the notion of filiform Lie superalgebras, generalizing the filiform Lie algebras studied by Vergne in the sixties. In these appers, the superalgebras whose even part is isomorphic to the model filiform Lie algebra $L_{n}$ are studied and classified in low dimensions. Here we consider a class of superalgebras whose even part is the filiform, naturally graded Lie algebra $Q_{n}$, which only exists in even dimension as a consequence of the centralizer property. Certain central extensions of $Q_{n}$ which preserve both the nilindex and the cited property are also generalized to obtain nonfiliform Lie superalgebras.
\end{abstract}

\section{Introduction}

Nilpotent Lie superalgebras are a relatively recent research field within the Lie superalgebras. The interest on solvable superalgebras increased in the 80's, when Bakhturin and Drenski [1] studied identities on solvable colored Lie superalgebras. Its representation have also been analyzed by various authors [11]. But there are very few works entirely devoted to nilpotent superlagebras. Up to dimension 5, they have been classified by Hegazi $([8],[9])$. Recently, the deformations of the standard filiform Lie superalgebra $L_{n, m}$ were determined [6], by estimating the dimensions of the cohomology groups $H_{0}^{2}\left(L_{n, m}, L_{n, m}\right)$.

In this work we are mainly interested in studying the equivalent structure, for the Lie superalgebras, of the nilpotent Lie algebra $Q_{n}$ determined by Vergne in 1966 [13]. It is well known that this algebra is

2000 Mathematics Subject Classification: 17B30, 17B70.

Servicio de Publicaciones. Universidad Complutense. Madrid, 2002 
characterized by its natural graduation and its nonexistence in odd dimension. This last property, which is a consequence of the structure of the ideals in the descending central sequence of $Q_{n}$, can be formulated in terms of centralizers, and has therefore been called the centralizer property. This can be used to extend Vergne's theorem [13] to wide classes of naturally graded Lie algebras. Now, for a nilpotent Lie superalgebra $G=G_{0} \oplus G_{1}$, this condition can be formulated also for the odd elements, and extended to a similar condition on the $G_{0}$-module $G_{1}$. This is done by starting from the associated Lie algebra to $G$, where $G_{0}=Q_{n}$ for $n \geq 6$.

\section{$1.1 \quad$ Generalities}

Definition 1. A Lie superalgebra is a $\mathbb{Z}_{2}$-graded vector space $G=$ $G_{0} \oplus G_{1}$ with a bilinear mapping [.,.] : $G \times G \leftarrow G$ satisfying

$$
\begin{aligned}
{\left[G_{\alpha}, G_{\beta}\right] } & \subset G_{\alpha+\beta} \\
{[X, Y] } & =-(-1)^{\operatorname{deg} X \operatorname{deg} Y}[Y, X] \\
{[X,[Y, Z]] } & =[[X, Y], Z]+(-1)^{\operatorname{deg} X \operatorname{deg} Y}[Y,[X, Z]]
\end{aligned}
$$

for all $X \in G_{\alpha}, Y \in G_{\beta}, z \in G$ and $\alpha, \beta \in \mathbb{Z}_{2}$

It follows from this definition that $G_{0}$ is an usual Lie algebra, that $G_{1}$ is an $G_{0}$-module and that there exists a $G_{0}$-invariant symmetric map $\rho: \bigvee^{2} G_{1} \rightarrow G_{0}$.

For an arbitrary Lie superlagebra we can also define a descending central sequence as follows :

$$
\begin{aligned}
& C^{0}(G)=G \\
& C^{k}(G)=\left[G, C^{k-1}(G)\right], k \geq 1
\end{aligned}
$$

Definition 2. A Lie superalgebra is called nilpotent if there exists a positive integer $n$ such that $C^{n}(G)=0$.

This definition can be modified to isolate the aportation of the Lie algebra of even elements and the module to the nilpotence of the structure. This can be reformulated as done in $[6,7]$ :

$$
C^{0} G_{0}=G_{0}, C^{k} G_{0}=\left[G_{0}, C^{k-1} G_{0}\right], k \geq 1
$$


which is nothing than the usual central descending sequence for $G_{0}$, and

$$
C^{0} G_{1}=G_{1}, C^{k}=\left[G_{0}, C^{k-1} G_{1}\right]=a d_{G} G_{0}\left(C^{k-1} G_{1}\right), k \geq 1
$$

Lemma 1. The Lie superalgebra $G=G_{0} \oplus G_{1}$ is nilpotent if and only if there exist positive integers $p, q$ such that

$$
C^{p} G_{0}=C^{q} G_{1}=\{0\}
$$

The proof is a reformulation of Engel's theorem.

Definition 3. The nilindex of the nilpotent Lie superlagebra $G=G_{0} \oplus$ $G_{1}$ is the minimal pair of integers $(p, q)$ that satisfy $C^{p} G_{0}=C^{q} G_{1}=$ $\{0\}$.

Recall that a nilpotent Lie algebra $\mathfrak{g}$ is called filiform if its nilindex is $p=\operatorname{dim} \mathfrak{g}-1$. Thus a Lie superalgebra $G=G_{0} \oplus G_{1}$ will be called filiform if $(p, q)=\left(\operatorname{dim} G_{0}-1, \operatorname{dim} G_{1}\right)$. As done for the Lie algebras, the filiform Lie superalgebras also define a variety whose properties are similar to those of usual Lie algebras. In [7], the author concentrates on the study of the filiform Lie superalgebra $L_{n, m}$ and its cohomology spaces, starting from the classical results known for the filiform model Lie algebra $L_{n}$. Here we focus on the algebra $Q_{n}$ and search for its equivalent in the variety of Lie superalgebras.

Recall that Vergne proved the following result:

Lemma 2. A naturally graded nilpotent Lie algebra is filiform if and only if it is isomorphic to one of the following algebras :

1. $L_{n}(n \geq 3)$ :

$$
\left[X_{1}, X_{i}\right]=X_{i+1}, 2 \leq i \leq n
$$

over the basis $\left\{X_{1}, . ., X_{n+1}\right\}$.

2. $Q_{2 m}(m \geq 3)$ :

$$
\begin{aligned}
{\left[X_{1}, X_{i}\right] } & =X_{i+1}, 2 \leq i \leq 2 m-1 \\
{\left[X_{j}, X_{2 m+1-j}\right] } & =(-1)^{j} X_{2 m}, 2 \leq j \leq m
\end{aligned}
$$

over the basis $\left\{X_{1}, . ., X_{2 m}\right\}$. 


\subsection{The centralizer property}

In this paragraph we recall the centralizer property for Lie algebras [4], which will be later be generalized for Lie superalgebras :

Definition 4. A naturally graded nilpotent Lie algebra $\mathfrak{g}$ is said to satisfy the centralizer property if

$$
\begin{array}{lll}
C_{\mathfrak{g}}\left(C^{p} \mathfrak{g}\right) & \supseteq \quad C^{p} \mathfrak{g}, p \geq\left[\frac{n(\mathfrak{g})}{2}\right] \\
C_{\mathfrak{g}}\left(C^{p} \mathfrak{g}\right) & \nsupseteq \quad C^{p} \mathfrak{g}, p<\left[\frac{n(\mathfrak{g})}{2}\right]
\end{array}
$$

where $n(\mathfrak{g})$ is the nilpotence class (or nilindex) of $\mathfrak{g}$.

Remark 1. For the algebra $Q_{n}$, the property gives an estimation of how far the algebra $Q_{n}$ is from having an abelian commutator algebra, as happens for $L_{n}$.

Let $G=G_{0} \oplus G_{1}$ be a Lie superalgebra. Then the associated Lie algebra is defined as follows : if [.,.] denotes the superalgebra product, we define the Lie algebra law $\mu$ as

1. $\mu(X, Y)=[X, Y]$ for any $X, Y \in G_{0}$

2. $\mu(X, Y)=[X, Y]$ for $X \in G_{0}, Y \in G_{1}$

3. $\mu(X, Y)=0$ for any $X, Y \in G_{1}$

This is nothing as considering the odd part as an abelian ideal. It is obvious that the associated Lie algebra, which is usually denoted by $\widetilde{G}$, is an invariant of the superalgebra.

Let $G_{0}$ be a nilpotent Lie algebra of nilindex $p$ and $G=G_{0} \oplus G_{1}$ a nilpotent Lie superalgebra. For $1 \leq r \leq \operatorname{dim} G_{1}$ we define the annihilator $A s s_{G_{1}}\left(C^{r} G_{1}\right)$ of $C^{r} G_{1}$ in $G_{1}$ as :

$$
A s s_{G_{1}}\left(C^{r} G_{1}\right)=\left\{X \in G_{1} \mid\left[X, C^{r} G_{1}\right]=0\right\}
$$

It can be immediately verified that it carries a structure of $G_{0}$-submodule of $G_{1}$. 
Definition 5. The Lie superalgebra $G$ is said to satisfy the centralizerannihilator property, shortened $C$ - $A$, if

1. $G_{0}$ satisfies the centralizer property as Lie algebra.

2. For $1 \leq q \leq \operatorname{dim} G_{1}$

$$
\begin{aligned}
& A s s_{G_{1}}\left(C^{q} G_{1}\right) \supseteq C^{q} G_{1} \text { for } q \geq\left[\frac{p}{2}\right] \\
& A s s_{G_{1}}\left(C^{q} G_{1}\right) \nsupseteq C^{q} G_{1} \text { for } q<\left[\frac{p}{2}\right]
\end{aligned}
$$

where $p$ is the nilindex of $G_{0}$.

3. The associated Lie algebra $\widetilde{G}$ is naturally graded.

Although the third requirement is not indispensable, it is rather convenient to consider the naturally graded Lie algebras, as deformation theory provides then the non graded models. On the other hand, the centralzer property for Lie algebras was originaly restricted to graded Lie algebras [4].

In particular this definition that the Lie algebra of even elements is naturally graded and that the dimension of $G_{1}$ is at least $\left[\frac{p}{2}\right]$.

\section{The Lie superalgebra $Q(2 m, 2 m)$}

In this section we construct a Lie superalgebra that constitutes, for the variety of Lie superalgebra laws, the natural generalization of the filiform Lie algebra $Q_{n}$.

For $m \geq 4$ let $\widetilde{Q}(2 m, 2 m)$ be the $4 m$-dimensional Lie algebra whose brackets over the basis $\left\{X_{1}, . ., X_{2 m}, Y_{1}, . ., Y_{2 m}\right\}$ are given by

$$
\begin{aligned}
{\left[X_{1}, X_{i}\right] } & =X_{i+1}, 2 \leq i \leq 2 m-1 \\
{\left[X_{j}, X_{2 m+1-j}\right] } & =(-1)^{j} X_{2 m}, 2 \leq j \leq m \\
{\left[X_{1}, Y_{i}\right] } & =Y_{i+1}, 1 \leq i \leq 2 m-1 \\
{\left[X_{2}, Y_{i}\right] } & =Y_{i+1}, 1 \leq i \leq 2 m-1
\end{aligned}
$$

In fact, the brackets $\left[X_{2}, Y_{i}\right]=Y_{i+1}$ could be ommited by an elementary change of basis. 
Lemma 3. For $m \geq 4$ the Lie algebra $\widetilde{Q}(2 m, 2 m)$ is naturally graded and nilpotent of nilindex $2 m$. Moreover

1. the subspace $V_{0}$ generated by $X_{1}, . ., X_{2 m}$ is a subalgebra isomorphic to $Q_{2 m}$

2. the subspace $V_{1}$ generated by the $Y_{1}, . ., Y_{2 m}$ is an abelian ideal.

3. for $q \geq m-1$

$$
C_{\widetilde{Q}(2 m, 2 m)} C^{q} \widetilde{Q}(2 m, 2 m) \supset C^{q} \widetilde{Q}(2 m, 2 m)
$$

The proof is elementary. Observe however that by 3$)$, the algebra $\widetilde{Q}(2 m, 2 m)$ does not satisfy the centralizer property. The next step is to find a filiform $\mathbb{Z}$-graded Lie superalgebra whose associated Lie algebra is isomorphic to $\widetilde{Q}(2 m, 2 m)$.

Definition 6. Let $G=G_{0} \oplus G_{1}$ be a Lie superalgebra, $\widetilde{G}$ its associated Lie algebra and $\mathfrak{g r}(\widetilde{G})=\oplus_{i \geq 1} \widetilde{G}_{i}$ the graded algebra related to $\widetilde{G}$, where $\widetilde{G_{i}}=\frac{C^{i-1} \widetilde{G}}{C^{i} \widetilde{G}}$ for $i \geq 1$. Then $G$ is called $\widetilde{G}$-compatible if the symmetric map $\rho: G_{1} \bigvee G_{1} \rightarrow G_{0}$ satisfies

$$
\rho\left(\widetilde{G_{i}}, \widetilde{G_{j}}\right) \subset \widetilde{G}_{i+j}, i, j \geq 1
$$

Theorem 1. There exists a unique $\mathbb{Z}$-graded $\widetilde{Q}(2 m, 2 m)$-compatible filiform Lie superalgebra $G=G_{0} \oplus G_{1}$ whose even part is isomorphic to $Q_{2 m}$ and for which $\rho$ is nontrivial. Over a basis $\left\{X_{1}, . ., X_{2 m}, Y_{1}, . ., Y_{2 m}\right\}$ its law is given by

$$
\begin{aligned}
{\left[X_{1}, X_{i}\right] } & =X_{i+1}, 2 \leq i \leq 2 m-1 \\
{\left[X_{j}, X_{2 m+1-j}\right] } & =(-1)^{j} X_{2 m}, 2 \leq j \leq m \\
{\left[X_{1}, Y_{i}\right] } & =Y_{i+1}, 1 \leq i \leq 2 m-1 \\
{\left[X_{2}, Y_{i}\right] } & =Y_{i+1}, 1 \leq i \leq 2 m-1 \\
{\left[Y_{i}, Y_{2 m-1+i}\right] } & =2(-1)^{m-1+i} X_{2 m}, 1 \leq i \leq m-1 \\
{\left[Y_{i}, Y_{2 m-2+i}\right] } & =(-1)^{m-1+i}(2 m-2 i-1) X_{2 m-1}, 1 \leq i \leq m-1
\end{aligned}
$$


Proof. From the associated Lie algebra $\widetilde{Q}(2 m, 2 m)$ we deduce the existence of a basis $\left\{X_{1}, . ., X_{2 m}, Y_{1}, . ., Y_{2 m}\right\}$ of $G$ such that $\left\{X_{1}, . ., X_{2 m}\right\}$ is a basis of $G_{0},\left\{Y_{1}, . ., Y_{2 m}\right\}$ is a basis of $G_{1}$ and the brackets relative to the $X_{i}$ and the module action are

$$
\begin{aligned}
{\left[X_{1}, X_{i}\right] } & =X_{i+1}, 2 \leq i \leq 2 m-1 \\
{\left[X_{j}, X_{2 m+1-j}\right] } & =(-1)^{j} X_{2 m}, 2 \leq j \leq m \\
{\left[X_{1}, Y_{i}\right] } & =Y_{i+1}, 1 \leq i \leq 2 m-1 \\
{\left[X_{2}, Y_{i}\right] } & =Y_{i+1}, 1 \leq i \leq 2 m-1
\end{aligned}
$$

Thus $G$ is $\widetilde{Q}(2 m, 2 m)$-compatible by taking the $\mathbb{Z}$-graduation defined by

$$
G_{(i)}=\left\{\begin{array}{c}
\left\langle X_{1}, X_{2}, Y_{1}\right\rangle \text { if } i=1 \\
\left\langle X_{i+1}, Y_{i}\right\rangle \text { if } 2 \leq i \leq 2 m-1 \\
\left\langle Y_{2 m}\right\rangle \text { if } i=2 m
\end{array}\right.
$$

As $a d_{G} X_{j}\left(G_{1}\right)=0$ if $j \geq 3$, it follows that the multiplication on $G_{1}$ is given by

$$
\left[Y_{i}, Y_{j}\right]=\alpha_{i j} X_{i+j+1}
$$

Now the product $\left[X_{2},\left[Y_{i}, Y_{j}\right]\right]$ on $G$ is zero unless $i+j=2 m-1$, which combined with the application of the Jacobi superidentity to the triples $\left\{X_{1}, Y_{i}, Y_{j}\right\}$ shows that $\alpha_{i j}=0$ for $i+j \leq 2 m-3$. This implies in particular that $a d_{G} Y_{i}\left(G_{0}\right) \in \mathbb{C} X_{2 m-1}+\mathbb{C} X_{2 m}$ for any $j$, so that Jacobi is satisfied automatically for all triples $\left\{Y_{i}, Y_{j}, Y_{k}\right\}$ and $\left\{Y_{i}, X_{j}, X_{k}\right\}$, where $a_{21}=1$ when applied to $j=1, k=2$. As we have

$$
\left[X_{i},\left[Y_{j}, Y_{k}\right]\right]=\left\{\begin{array}{cc}
\alpha_{j k} X_{2 m}, & i=1,2,(j, k)=(m-k, m+k) \text { for } k \geq 1 \\
0 & i \geq 3, j=1, . ., 2 m
\end{array}\right.
$$

we obtain succesively the relations

1. $\alpha_{m-1, m-1}=2 \alpha_{m-1, m}$ for the triple $\left\{X_{1}, Y_{m-1}, Y_{m-1}\right\}$

2. $\alpha_{m-1-k, m-1+k}=\alpha_{m-1-k, m+k}+\alpha_{m-k, m-1+k}$ for the triples $\left\{X_{1}, Y_{m-1-k}, Y_{m-1+k}\right\}$ and $1 \leq k \leq m-2$

3. $0=\alpha_{m-1-k, m-1+k}+\alpha_{m-k, m-2-k}$ for the triples $\left\{X_{1}, Y_{m-1-k}, Y_{m-2-k}\right\}$ and $0 \leq k \leq m-2$ 
Thus all nonzero structure constants $\alpha_{i j}$ are a multiple of $\alpha_{m-1, m}$. It follows

$$
\begin{aligned}
\alpha_{i, 2 m-2-i} & =2(-1)^{m-1+i} \alpha_{m-1, m}, 1 \leq i \leq m-1 \\
\alpha_{i, 2 m-1-i} & =(-1)^{m-1+i}(2 m-2 i-1) \alpha_{m-1, m}, 1 \leq i \leq m-1
\end{aligned}
$$

A change of basis $Y_{1}^{\prime}=\beta Y_{1}$ with $\beta^{2} \alpha_{m-1, m}=1$ allows to suppose $\alpha_{m-1, m}=1$, from which the Lie superalgebra law follows.

We will denote this superalgebra by $Q(2 m, 2 m)=Q_{2 m} \oplus Q_{1}(2 m, 2 m)$

Corollary 1. The Lie superlagebra $Q(2 m, 2 m)$ satisfies the centralizerannihilator property.

Proof. For the subalgebra of even elements the situation is obvious. Now

$$
C^{j} Q_{1}(2 m, 2 m)=\left\langle Y_{j+1}, . ., Y_{2 m}\right\rangle, j \geq 1
$$

As $Y_{m-1} \in C^{m-2} Q_{1}(2 m, 2 m)-C^{m-1} Q_{1}(2 m, 2 m)$, we obtain clearly the annihilator property.

Remark 2. Observe that the C-A property for $Q(2 m, 2 m)$ is a consequence of the nonnullity of $\rho$.

The space of even derivations of $Q(2 m, 2 m)$ is denoted by $\operatorname{Der}_{0}(Q(2 m, 2 m))$, as usual [12]. If $f$ is such a derivation, then we write

$$
\begin{gathered}
f\left(X_{i}\right)=f_{i}^{j} X_{j}, 1 \leq i \leq 2 m \\
f\left(Y_{i}\right)=g_{i}^{j} Y_{j}, 1 \leq i \leq 2 m
\end{gathered}
$$

As $\left.f\right|_{Q_{0}(2 m, 2 m)}$ is a derivation of $Q_{2 m}$, it is well known that

$$
\begin{aligned}
f_{i}^{j} & =0, i \geq 1, j<i ; f_{1}^{2}=f_{2}^{2}-f_{1}^{1} ; f_{2}^{4}=0 \\
f_{i}^{i} & =(i-2) f_{1}^{1}+f_{2}^{2} \text { for } 3 \leq i \leq 2 m-1 ; f_{2 m}^{2 m}=(2 m-2) f_{1}^{1}+2 f_{2}^{2} ; \\
f_{i}^{i+k} & =f_{2}^{i+k-1} \text { for } i \geq 3,1 \leq k \leq 2 m-i-1 ; \\
f_{i}^{2 m} & =f_{2}^{2 m+2-i}+(-1)^{i} f_{1}^{2 m+2-i} \text { for } i<2 m
\end{aligned}
$$


From the conditions $\left[f\left(X_{1}\right), Y_{i}\right]+\left[X_{1}, f\left(Y_{i}\right)\right]=f\left(Y_{i+1}\right), 1 \leq i \leq 2 m-1$ we obtain then

$$
\begin{aligned}
g_{k}^{k} & =(k-1) f_{2}^{2}+g_{1}^{1} \\
g_{k}^{j} & =g_{1}^{j-k}, k+1 \leq j \leq 2 m \\
g_{k}^{j} & =0 \text { for } j<k
\end{aligned}
$$

Now, for $1 \leq i \leq m-2$ we have $\left[Y_{i}, Y_{i}\right]=0$, thus we obtain $2\left[f\left(Y_{i}\right), Y_{i}\right]=0=(-1)^{m-1-i} g_{i}^{2 m-2-i} X_{2 m-1}+(-1)^{m-1-i} g_{i}^{2 m-1-i} X_{2 m}$

and therefore $g_{1}^{j}=0$ for $3 \leq j \leq 2 m-3$. Finally, from the nontrivial brackets $\left[f\left(Y_{1}\right), Y_{2 m-3}\right]+\left[Y_{1}, f\left(Y_{2 m-3}\right)\right]=2(-1)^{m} f\left(X_{2 m-1}\right)$ we deduce

$$
\begin{aligned}
2 g_{1}^{1} & =(2 m-3) f_{1}^{1}-(2 m-5) f_{2}^{2} \\
g_{1}^{2} & =f_{2}^{3}-f_{1}^{3}
\end{aligned}
$$

The remaining possibilities give no more additional conditions, thus we resume

Proposition 1. For $m \geq 4$ the following holds

$$
\operatorname{dim} \operatorname{Der}_{0}(Q(2 m, 2 m))=4 m-1
$$

\section{$3 \quad$ The superalgebras $\mathfrak{g}_{(m, t)}(2 m)$}

In this section we construct, for $m \geq 4, m-2$ nilpotent Lie superalgebras whose even part is a central extension of $Q_{n}$ by $\mathbb{C}$. The interest of this construction is that both the dimension of the odd part and its structure is the same as for the superalgebra $Q(2 m, 2 m)$.

Recall that, for a Lie algebra $\mathfrak{g}$, the space $H^{2}(\mathfrak{g}, \mathbb{C})$ can be interpreted as the space of classes of 1-dimensional central extensions of the Lie algebra $\mathfrak{g}$. It is well known that the space of 2-cocycles $Z^{2}(\mathfrak{g}, \mathbb{C})$ is identified with the space of linear forms over $\bigwedge^{2} \mathfrak{g}$ which are zero over the subspace $\Omega$ :

$$
\Omega:=\left\langle\mu_{0}(x, y) \wedge z+\mu_{0}(y, z) \wedge x+\mu_{0}(z, x) \wedge y\right\rangle_{\mathbb{C}}
$$


The extension classes are defined modulus the coboundaries $B^{2}(\mathfrak{g}, \mathbb{C})$. This allows to identify the cohomology space $H^{2}(\mathfrak{g}, \mathbb{C})$ with the dual of the space $\frac{\operatorname{Ker} \lambda}{\Omega}$, where $\lambda \in \operatorname{Hom}\left(\Lambda^{2} \mathfrak{g}, \mathfrak{g}\right)$ is defined as

$$
\lambda(x \wedge y)=\mu_{0}(x, y) \quad x, y \in \mathfrak{g}
$$

In fact we have $H_{2}(\mathfrak{g}, \mathbb{C})=\frac{K e r \lambda}{\Omega}$ for the 2-homology space, and as $H^{2}(\mathfrak{g}, \mathbb{C})=\operatorname{Hom}_{\mathbb{C}}\left(H_{2}(\mathfrak{g}, \mathbb{C}), \mathbb{C}\right)$ the assertion follows.

Notation 1. Let $\psi_{i j} \in C^{2}(\mathfrak{g}, \mathbb{C})$ the cocycles defined by

$$
\psi_{i j}\left(X_{k}, X_{l}\right)=\delta_{i k} \delta_{j l}
$$

Thus any cocycle can be expressed as a linear combination of the preceding cochains.

Notation 2. For $k \geq 2$ let

$$
H_{k}^{2, t}(\mathfrak{g}, \mathbb{C})=\left\{\varphi_{i j} \in H^{2}(\mathfrak{g}, \mathbb{C}) \mid \varphi_{i j}\left(\mathfrak{g}_{k}, \mathfrak{g}_{l}\right) \subset \delta_{i k} \delta_{j l} \mathfrak{g}_{2 t+k+1}\right\},
$$

$1 \leq t \leq\left[\frac{n-3}{2}\right]$, where $\mathfrak{g}=\sum_{k \geq 1} \mathfrak{g}_{k}$ and $\mathfrak{g}_{k}=\frac{C^{k-1} \mathfrak{g}}{C^{k} \mathfrak{g}}$ for $k \geq 1$.

Let $m \geq 4$ and $\mathfrak{g}_{(m, t)} \quad(1 \leq t \leq m-2)$ be the Lie algebra whose Cartan-Maurer equations over the basis $\left\{\omega_{1}, . ., \omega_{2 m+1}\right\}$ are :

$$
\begin{aligned}
d \omega_{1} & =d \omega_{2}=0 \\
d \omega_{j} & =\omega_{1} \wedge \omega_{j-1}, 3 \leq j \leq 2 m-1 \\
d \omega_{2 m} & =\omega_{1} \wedge \omega_{2 m-1}+\sum_{j=2}^{\left[\frac{2 m+1}{2}\right]}(-1)^{j} \omega_{j} \wedge \omega_{2 m+1-j} \\
d \omega_{2 m+1} & =\sum_{j=2}^{t+1}(-1)^{j} \omega_{j} \wedge \omega_{3-j+2 t}
\end{aligned}
$$

Proposition 2. For $m \geq 4$ the Lie algebra $\mathfrak{g}_{(m, t)}$ is a central extension of $Q_{2 m}$ by $\mathbb{C}$ that preserves the natural graduation, the nilindex and the centralizer property.

Proof. A central extension of $Q_{2 m}$ by $\mathbb{C}$ which satisfies the required conditions is easily seen to be determined by the cocycles $\varphi_{i j} \in H_{2}^{2, t}\left(Q_{2 m}, \mathbb{C}\right)$ 
for $1 \leq t \leq m-2$. Now these cocycles must satisfy the relation $i+j=2 t+3$. It is immediate to verify that this space is generated by the cocycles

$$
\varphi_{2,2 t+1}, \varphi_{3,2 t}, \ldots, \varphi_{t+1, t+2}
$$

subjected to the relations

$$
\varphi_{2,2 t+1}+(-1)^{j-1} \varphi_{j, 2 t+3-j}=0, j=3, . ., t+1
$$

If $\left\{X_{1}, . ., X_{2 m}\right\}$ is the dual base of $\left\{\omega_{1}, . ., \omega_{2 m}\right\}$, we have

$$
X_{2,2 t+1}, X_{3,2 t}, \ldots, X_{t+1, t+2} \in \operatorname{Ker} \lambda
$$

and

$$
X_{2,2 t+1}+(-1)^{j-1} X_{j, 2 t+3-j} \in \Omega, j=3, . ., t+1
$$

Thus there is, for any $t$, only one extension, which is isomorphic to $\mathfrak{g}_{(m, t)}$. For the remaining values of $t$ it is easy to see that $Q_{2 m}$ does not admit naturally graded extensions with the prescribed properties.

Remark 3. The preceding family are the only extensions of $Q_{n}$ that satisfy the centralizer property and preserve both the nilindex and the natural graduation. In [4] the $(2 m+1)$-dimensional naturally graded Lie algebras of nilindex $2 m-1$ which satisfy the property are classified.

Let $G=G_{0} \oplus G_{1}$, where $G_{0}=\mathfrak{g}_{(m, t)}$ for $1 \leq t \leq m-2$ and $G_{1}=$ $\bigoplus_{i=1}^{2 m} \mathbb{C} Y_{i}$. Define a $G_{0}$-module structure on $G_{1}$ by

$$
\left[X_{i}, Y_{j}\right]=Y_{j+1}, i=1,2,1 \leq j \leq 2 m-1,
$$

and the symmetric map $\rho: G_{1} \vee G_{1} \rightarrow G_{0}$

$$
\begin{array}{r}
\rho\left(Y_{i}, Y_{2 m-2-i}\right)=2(-1)^{m+i-1} X_{2 m-1}, 1 \leq i \leq m-1 \\
\rho\left(Y_{i}, Y_{2 m-1-i}\right)=(2 m-2 j-1)(-1)^{m+i-1} X_{2 m}, 1 \leq i \leq m-1
\end{array}
$$

This map is a cocycle and can also be deduced from [6].

Theorem 2. For $m \geq 4$ and $1 \leq t \leq m-2$ the sum $G_{0} \oplus G_{1}$ is a nilpotent Lie superalgebra that satisfies the centralizer-annihilator property. 
Proof. Observe that $a d\left(Y_{i}\right)\left(G_{1}\right) \in \mathbb{C} X_{2 m-1}+\mathbb{C} X_{2 m}$ for any $i$, so that the Jacobi superidentity is satisfied for all triples $\left\{Y_{i}, Y_{j}, Y_{k}\right\}$. As $X_{1}, X_{2} \notin C^{1} G_{0}$ and $\left[X_{i}, G_{1}\right]=0$ for $i \geq 3$, the triples $\left\{X_{i}, X_{j}, Y_{k}\right\}$ also are satisfied. Finally, for the triples $\left\{X_{i}, Y_{j}, Y_{k}\right\}$ for $i=1,2$ we recover the coefficient relations expressed by $\rho$.

Remark 4. The preceding Lie superalgebras will be denoted by $\mathfrak{g}_{(m, t)}(2 m)$. Observe that the odd elements are the same as for the superalgebra $Q(2 m, 2 m)$, and the adjoined vector does not alter the product of $G_{1}$. This occurs whenever the nilindex of the even part is not altered. In particular, its even derivations will be similar to those of $Q(2 m, 2 m)$, as the intervention of $X_{2 m+1}$ is void.

\subsection{The superalgebras $\mathfrak{g}_{(m, t)}^{1, q}(2 m)$}

If the procedure of the preceding section is generalized, we obtain also Lie superalgebras whose even part is obtained by extending centrally, under certain conditions, the filiform algebra $Q_{2 m}$.

For $m \geq 4,1 \leq t \leq m-2$ and $0 \leq q \leq 2 m-2 t-3$ let $\mathfrak{g}_{(m, t)}^{1, q}$ be the Lie algebra whose Cartan-Maurer equations over the basis $\left\{\omega_{1}, . ., \omega_{2 m+1}, . ., \omega_{2 m+2+q}\right\}$ are

$$
\begin{aligned}
d \omega_{1} & =d \omega_{2}=0 \\
d \omega_{j} & =\omega_{1} \wedge \omega_{j-1}, 3 \leq j \leq 2 m-1 \\
d \omega_{2 m} & =\omega_{1} \wedge \omega_{2 m-1}+\sum_{j=2}^{\left[\frac{2 m+1}{2}\right]}(-1)^{j} \omega_{j} \wedge \omega_{2 m+1-j} \\
d \omega_{2 m+1} & =\sum_{j=2}^{t+1}(-1)^{j} \omega_{j} \wedge \omega_{3-j+2 t} \\
d \omega_{2 m+2} & =\omega_{1} \wedge \omega_{2 m+1}+\sum_{j=2}^{t+1}(-1)^{j}(t+2-j) \omega_{j} \wedge \omega_{4-j+2 t} \\
d \omega_{2 m+2+r} & =\omega_{1} \wedge \omega_{2 m+1+r}+\sum_{j=2}^{t+1}(-1)^{j} S_{j}^{r} \omega_{j} \wedge \omega_{4-j+2 t+r}, 1 \leq r \leq q
\end{aligned}
$$


where

$$
\begin{aligned}
& S_{j}^{1}=\sum_{k=j}^{t+1}(t+2-k), 2 \leq j \leq t+1 \\
& S_{j}^{k}=\sum_{k=j}^{t+1} S_{j}^{k-1}, \quad 2 \leq k \leq q
\end{aligned}
$$

Proposition 3. For any $m \geq 4$ the Lie algebra $\mathfrak{g}_{(m, t)}^{1, q}$ is a central extension of $\mathfrak{g}_{(m, t)}^{1, q-1}$ by $\mathbb{C}$ which satisfies the centralizer property.

Proof. We first prove that $\mathfrak{g}_{(m, t)}^{1,0}$ is an extension of $\mathfrak{g}_{(m, t)}$. For this, the cocycles which define an extension with the required property are

$$
\varphi_{j, 4-j+2 t} \in H_{2}^{2, \frac{2 t+1}{2}}\left(\mathfrak{g}_{(m, t)}^{2}, \mathbb{C}\right), \varphi_{1,2 m+1} \in H_{2 m-2 t}^{2, \frac{2 t+1}{2}}\left(\mathfrak{g}_{(m, t)}^{2}, \mathbb{C}\right)
$$

satisfying

$$
\begin{aligned}
\varphi_{1,2 m+1}+\varphi_{t+1,3+t} & =0 \\
\varphi_{2,2+t}+(-1)^{j}(t+2-j) \varphi_{j, 4-j+2 t} & =0,3 \leq j \leq t+1
\end{aligned}
$$

For the general case recall that for $\mathfrak{g}_{(m, t)}^{1}$ the last differential form is given by

$$
d \omega_{2 m+2}=\omega_{1} \wedge \omega_{2 m+1}+\sum_{j=2}^{t+1}(-1)^{j}(t+2-j) \omega_{j} \wedge \omega_{4-j+2 t}
$$

A central extension of $\mathfrak{g}_{(m, t)}^{1}$ by $\mathbb{C}$ which satisfies the centralizer property will be determined by the adjunction of a differential form $d \omega_{2 m+3}$, whose structure is

$$
d \omega_{2 m+3}=\omega_{1} \wedge \omega_{2 m+2}+\sum_{j=2}^{t+1}(-1)^{j} \varphi_{j, 5-j+2 t} \omega_{j} \wedge \omega_{5-j+2 t},
$$

where the cocycles

$$
\varphi_{j, 5-j+2 t} \in H_{2}^{2, t+1}\left(\mathfrak{g}_{(m, t)}^{1}, \mathbb{C}\right)
$$


satisfy

$$
\varphi_{2,3+2 t}+(-1)^{j} \sum_{k=j}^{t+1}(t+2-k) \varphi_{j, 5-j+2 t}=0,3 \leq j \leq t+1
$$

We thus obtain a unique extension class which is isomorphic to $\mathfrak{g}_{(m, t)}^{1,1}$. This shows the assertion for $q=1$. Let it be true for $q_{0}>1$. Then the Cartan-Maurer equations of $\mathfrak{g}_{(m, t)}^{1, q_{0}}$ are

$$
\begin{aligned}
d \omega_{1} & =d \omega_{2}=0 \\
d \omega_{j} & =\omega_{1} \wedge \omega_{j-1}, \quad 3 \leq j \leq 2 m-1 \\
d \omega_{2 m} & =\omega_{1} \wedge \omega_{2 m-1}+\sum_{j=2}^{\left[\frac{2 m+1}{2}\right]}(-1)^{j} \omega_{j} \wedge \omega_{2 m+1-j} \\
d \omega_{2 m+1} & =\sum_{j=2}^{t+1}(-1)^{j} \omega_{j} \wedge \omega_{3-j+2 t} \\
d \omega_{2 m+2} & =\omega_{1} \wedge \omega_{2 m+1}+\sum_{j=2}^{t+1}(-1)^{j}(t+2-j) \omega_{j} \wedge \omega_{4-j+2 t} \\
d \omega_{2 m+2+r} & =\omega_{1} \wedge \omega_{2 m+1+r}+\sum_{j=2}^{t+1}(-1)^{j} S_{j}^{r} \omega_{j} \wedge \omega_{4-j+2 t+r}, 1 \leq r \leq q_{0}
\end{aligned}
$$

Now we extend this algebra by $\mathbb{C}$. Supposing that the extension satisfies the centralizer property and is naturally graded of the prescribed characteristic sequence, the determining cocycles are

$$
\begin{aligned}
& \varphi_{j, 4-j+2 t+q_{0}+1} \in H_{2}^{2, \frac{2 t+2+r}{2}}\left(\mathfrak{g}_{(m, t)}^{1, q_{0}}, \mathbb{C}\right) \text { if } r \equiv 1(\bmod 2) \\
& \varphi_{j, 4-j+2 t+q_{0}+1} \in H_{2}^{2, t+\frac{r}{2}+1}\left(\mathfrak{g}_{(m, t)}^{1, q_{0}}, \mathbb{C}\right) \text { if } r \equiv 0(\bmod 2)
\end{aligned}
$$

We have the relations

$$
\varphi_{j, 4-j+2 t+q_{0}+1}+(-1)^{j} \sum_{j=2}^{t+1} S_{j}^{q_{0}} \omega_{j} \wedge \omega_{5-j+2 t+q_{0}}=0,3 \leq j \leq t+1
$$


and by an elementary change of basis, the adjoined differential form $d \omega_{2 m+3+q_{0}}$ is of type

$$
d \omega_{2 m+3+q_{0}}=\omega_{1} \wedge \omega_{2 m+2+q_{0}}+\sum_{j=2}^{t+1}(-1)^{j} S_{j}^{q_{0}} \omega_{j} \wedge \omega_{5-j+2 t+q_{0}}
$$

Both the nilindex, graduation and centralizer property are obviously satisfied.

We denote by $\mathfrak{g}_{(m, t)}^{1, q}(2 m)$ the Lie superalgebra whose even part is isomorphic to $\mathfrak{g}_{(m, t)}^{1, q}$ and odd part $G_{1}=\bigoplus_{i=1}^{2 m} \mathbb{C} Y_{i}$ with the module structure

$$
\left[X_{i}, Y_{j}\right]=Y_{j+1} i=1,2,1 \leq i \leq 2 m-1,
$$

and the symmetric map $\rho: G_{1} \vee G_{1} \rightarrow G_{0}$

$$
\begin{array}{r}
\rho\left(Y_{i}, Y_{2 m-2-i}\right)=2(-1)^{m+i-1} X_{2 m-1}, 1 \leq i \leq m-1 \\
\rho\left(Y_{i}, Y_{2 m-1-i}\right)=(2 m-2 j-1)(-1)^{m+i-1} X_{2 m}, 1 \leq i \leq m-1
\end{array}
$$

Theorem 3. The Lie superalgebra $\mathfrak{g}_{(m, t)}^{1, q}(2 m)$ is nilpotent of superindex $(2 m-1,2 m)$ and satisfies the centralizer-annihilator property.

Remark 5. Observe that these algebras, as well as $\mathfrak{g}_{(m, t)}(2 m)$, are not filiform any more. Thus the construction provides, starting from a filiform Lie superalgebra, family of nonfiliform Lie superalgbras in arbitrary dimension.

\section{References}

[1] Yu. A. Bakhturin, V. S. Drewnski, The identities of solvable colored Lie algebras, Algebra i Logika, 26 (1987), 403-418.

[2] K. Bauwens and L. Le Bruiyn, Some remarks on solvable Lie superalgebras, J. Pure Appl. Algebra, 99 (1995), 113-134.

[3] M. Boral, N. Ekizi and Y. Ünlü, On finitely generated free nilpotent Lie superalgebras of class <5, Algebras Groups Geom., 12 (1995), no. 3, 247254.

[4] R. Campoamor, J. M. Ancochea, On certain families of naturally graded Lie algebras, J. Pure Appl. Algebra, to appear. 
[5] L. Corwin, Y. Ne'eman and S. Sternberg, Graded Lie algebras in matehmatics and physics, Rev. Mod. Phys., 47 (1975), 573-603.

[6] M. Gilg, Superalgèbres de Lie nilpotentes, Ph. D. Thesis, Mulhouse 2000.

[7] M. Gilg, Low-dimensional filiform Lie superalgebras, Rev. Mat. Compl. XIV (2001), 463-478.

[8] A. Hegazi, Classification of nilotent Lie superalgebras of dimension five I, Internat. J. Theoret. Phys., 38 (1999), no. 6, 1735-1739.

[9] A. Hegazi, Classification of nilotent Lie superalgebras of dimension five II, Internat. J. Theoret. Phys., 38 (1999), no. 10, 2681-2693.

[10] A. Hegazi, A classification of nilotent Lie superalgebras of dimension five I, Panamer. Math. J., 10 (2000), no. 1, 75-93.

[11] V. G. Kac, Lie superalgbras, Adv. Math., 26 (1977), 8-96.

[12] M. Scheunert, The theory of Lie superlagebras, L.N.M 716 (1978).

[13] M. Vergne, Cohomologie des algèbres de Lie nilpotente. Applications a l'étude de la variété des algèbres de Lie nilpotentes, Bull. Soc. Math. France, 78 (1970), 81-116.

Departamento de Geometría y Topología Facultad CC. Matemáticas Univ. Complutense 28040 Madrid (Spain)

E-mail: Jose_Ancochea@mat.ucm.es

Departamento de Geometría y Topología

Facultad CC. Matemáticas Univ. Complutense 28040 Madrid (Spain)

E-mail: rutwig@nfssrv.mat.ucm.es

Recibido: 7 de Diciembre de 2000

Revisado: 11 de Mayo de 2001 